\title{
Interpretación del contenido de la humedad del suelo para determinar capacidad de campo y evitar riego excesivo en suelos arenosos utilizando sensores de humedad $^{1}$
}

\author{
Lincoln Zotarelli, Michael D. Dukes, y Kelly T. Morgan²
}

\section{Capacidad del suelo para almacenar agua}

Los suelos contienen diferente cantidad de agua dependiendo de su textura y estructura. El límite superior de almacenamiento de agua se denomina con frecuencia "capacidad de campo" (CC), mientras que el límite inferior se denomina "punto de marchitamiento permanente" (PMP). Después de un evento de lluvia o de riego que satura el suelo, hay un rápido movimiento descendente (drenaje) de una parte del agua del suelo debido a la fuerza de gravedad. Durante el proceso de drenaje, la humedad del suelo disminuye continuamente. La velocidad de drenaje está relacionada con la conductividad hidráulica del suelo. En otras palabras, el drenaje es más rápido en los suelos arenosos en comparación con los suelos arcillosos. Después de un tiempo, el rápido drenaje se hace insignificante y en ese punto, la humedad del suelo se denomina "capacidad de campo." El punto de marchitamiento permanente se define como el contenido de humedad del suelo en el que la planta ya no tiene la capacidad de absorber agua del suelo haciendo que la planta se marchite y muera si agua adicional no es proporcionada. Sin embargo, la mayoría de las plantas estarán sometidas a un estrés hídrico significativo antes de este punto, y será muy factible que las plantas sufran una reducción importante en su rendimiento mucho antes de alcanzar el punto de marchitamiento.

La cantidad total de agua disponible para absorción de la planta es el "agua disponible para la planta" (ADP) que es la diferencia entre CC y PMP (Figura 1) y a menudo se expresa como un porcentaje en volumen (volumen de agua/ volumen de muestra de suelo). La "capacidad de retención de agua disponible" (CRAD) se determina multiplicando el ADP por la profundidad de la zona de las raíces donde se produce la extracción de agua. La disminución del contenido de agua al PMP afecta negativamente la salud y el rendimiento de la planta. Así, para fines de riego, un "reducción máxima permisible" (RMP) o fracción del CRAD que representa el "agua disponible" para la planta, (AD) es el rango operativo ideal de contenido de agua del suelo para la planificación del riego. Teóricamente, la programación del riego consiste en iniciar el riego a bajos contenidos de agua de suelo, correspondientes al RMP dado, y regar hasta que el agua eliminada haya sido remplazada sin exceder

1. The English version of this document is AE460 - Interpretation of Soil Moisture Content to Determine Soil Field Capacity and Avoid Over-Irrigating Sandy Soils Using Soil Moisture Sensors. Este documento es AE496, uno de una serie del Departamento de Ingeniería Agrícola y Biológica, Servicio de Extensión Cooperativa, Instituto de Alimentos y Ciencias Agrícolas, Universidad de Florida. Traducio del Inglés por Rafael Arango. Publicado: Enero 2013. Visite el sitio web EDIS en http://edis.ifas.ufl.edu.

2. Lincoln Zotarelli, asistente de investigación científico; Michael D. Dukes, profesor asociado, Departamento de Ingeniería Agrícola y Biológica; Kelly T. Morgan, profesor asociado, Departamento de Ciencias del Suelo y del Agua; Servicio de Extensión Cooperativa, Instituto de Alimentos y Ciencias Agrícolas, Universidad de Florida, Gainesville, FL 32611. 
CC, de lo contrario, ocurriría una percolación o un drenaje profundo.

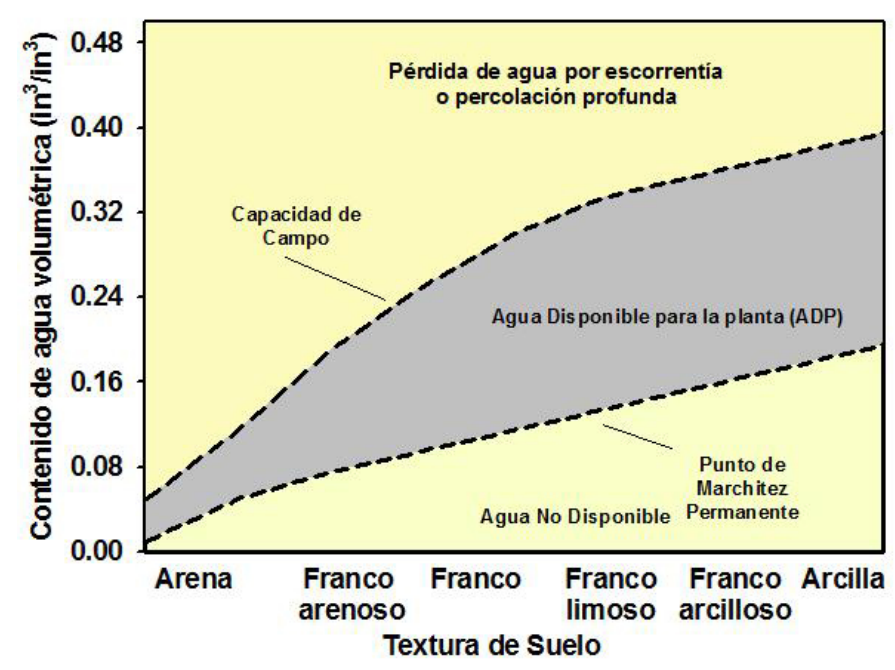

Figura 1. Relación general entre el agua disponible para la planta (ADP), la capacidad de campo, el punto de marchitez permanente, el agua no disponible y la clase de textura del suelo.

\section{Programación del riego}

La programación del riego es simplemente la aplicación de agua a los cultivos en el momento "adecuado" y en la cantidad "adecuada," y se considera una buena práctica de gestión (BPG). Por lo general, la programación del riego la establece la decisión del productor agrícola en la mayoría de los casos, o mediante el uso de un calendario predeterminado de programas de riego basado en las necesidades de agua de las campañas anteriores. Con el fin de establecer una adecuada programación del riego, varios factores son tomados en cuenta, tales como: la capacidad de evapotranspiración de la planta, las características del suelo y la distribución de las raíces. En Florida se usa una amplia gama de métodos para la programación de riego, con sus correspondientes niveles de aplicación del agua. El método recomendado para la programación de riego (por goteo o por aspersión) en cultivos de hortalizas es combinar, (1) las necesidades hídricas del cultivo teniendo en cuenta la etapa de crecimiento de la planta; (2) la medición del contenido de agua del suelo; y (3) las directrices para el riego dosificado.

Generalmente, existen dos tipos de sensores que se utilizan para la programación del riego; los que miden el potencial de agua del suelo (también llamado tensión o succión) y aquellos que miden el contenido de agua volumétrica directamente. Detalles sobre los sensores de humedad del suelo aparecen en la publicación, Field Devices for
Monitoring Soil Water Content (http://edis.ifas.ufl.edu/ ae266). La presente publicación se centra en dispositivos de medición de humedad del suelo basados en capacitancia, los cuales han mostrado una correcta medición de la humedad del suelo en los suelos arenosos comunes en Florida. Los sensores basados en capacitancia se han vuelto más populares en los últimos años debido a una disminución en el costo de los componentes electrónicos y una mayor fiabilidad.

Estos sensores de humedad del suelo (SHS), han sido exitosamente utilizados para controlar el contenido volumétrico de agua y orientar la programación del riego. Sin embargo, una correcta interpretación de las lecturas de humedad del suelo es muy importante para garantizar el manejo adecuado del riego y evitar riego excesivo riego.

\section{Determinación práctica de la capacidad de campo para suelos arenosos usando mediciones de humedad del suelo}

La Figura 2 muestra el contenido de humedad de suelo a una profundidad de 0-6 pulgadas, medidas por un sensor de capacitancia durante un período de 4 días. Hubo dos riegos los días 11/06 y 11/09 a las 7:00 am. Para la determinación del punto de capacidad de campo, aplicamos intencionalmente una cantidad de agua en el riego que llevo la capa de suelo estudiado a saturación, en este caso en particular, 0-6 pulgadas.

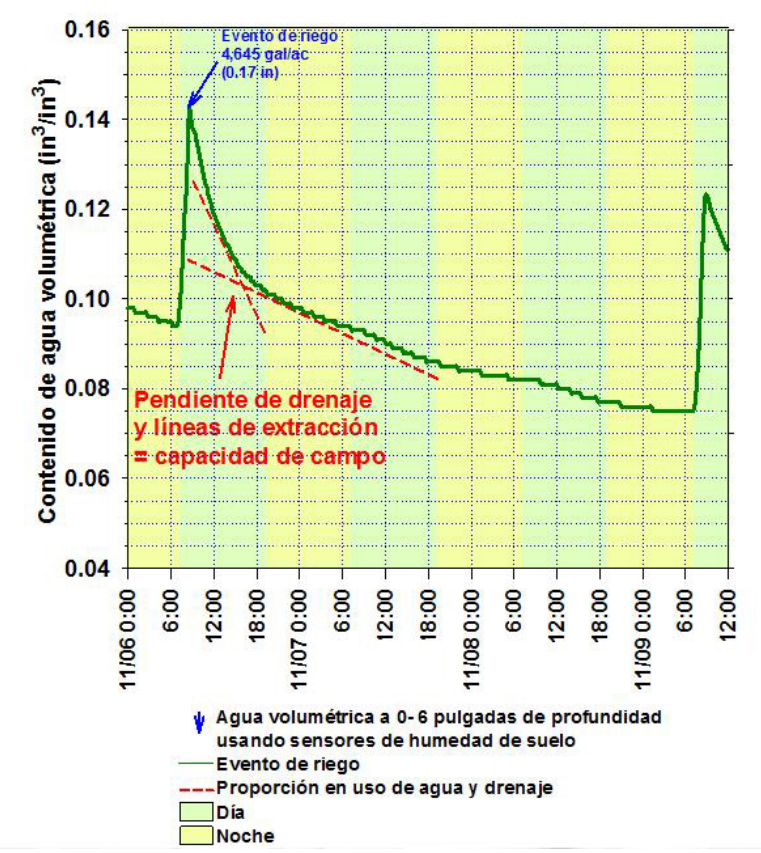

Figura 2. Ejemplo de determinación práctica de la capacidad de campo para un suelo arenoso tras un evento de riego. 
La profundidad de riego aplicada fue de 0.17 pulgadas (equivalentes a 4,645 gal/ac con riego por goteo). Después de los dos eventos de riego, se evidenció un aumento en el contenido de humedad del suelo. El grado al cual el contenido de humedad del suelo aumenta, sin embargo, depende del volumen de riego, el cual por lo general se establece por la duración del evento de riego. Para el riego por goteo con acolchado plástico en suelos arenosos, ciclos de riego largos, dan como resultado un gran aumento en la humedad del suelo en el área debajo del emisor de goteo. En la Figura 2, el pico de humedad del suelo es solamente temporal, en la medida que el agua de riego se drena rápidamente en profundidad más allá de las 6 pulgadas (11/06, entre las 9:00 am y 7:00 pm). Este aumento rápido en el contenido de agua, indica que el contenido de agua del suelo medido con los SHS rápidamente alcanza un punto por encima de la capacidad de retención de agua del suelo, y el agua percoló hacia las capas más profundas del suelo. Entre el 11/06 a las 6:00 pm y el 11/07 a las 7:00 pm (Figura 2), la tasa de disminución del contenido de agua del suelo mostró una tasa constante debido a la "lenta" extracción de agua por drenaje, que se produjo durante el día y la noche. La evapotranspiración, también disminuyó el contenido de agua del suelo, que tuvo lugar exclusivamente durante el día. Para suelos arenosos, el punto en el cual la pendiente del drenaje y las líneas de extracción cambian de una disminución de contenido de agua del suelo "rápida" a una "más lenta" se pueden asumir como el "punto de capacidad de campo." En este punto, el agua ha sido drenada a través de los poros grandes del suelo (macroporos), y ha sido reemplazada por aire. Los espacios porosos remanentes, (microporos), siguen todavía llenos de agua y le suministraran a las plantas la humedad requerida. Es muy importante que el operario de riego entienda este concepto de "capacidad de campo," para establecer una estrategia de control de riego con el fin último de suministrar una humedad de suelo óptima para el crecimiento de la planta, productividad, y reducir la pérdida de nutrientes por lixiviación.

\section{Ejemplos de la planificación de riego de cultivos hortícolas en suelos arenosos}

Las Figuras 3 y 4 muestran ejemplos de exceso deriego y de un manejo adecuado del riego por goteo, respectivamente. En la Figura 3, el riego se aplicó una vez por día con una profundidad de 0,18 pulgadas, equivalente a $4,718 \mathrm{gal} / \mathrm{ac}$ con riego por goteo. Después de cada evento de riego, se produjo un aumento en el contenido de humedad del suelo seguido por un drenaje rápido. Eventos de lluvia pueden

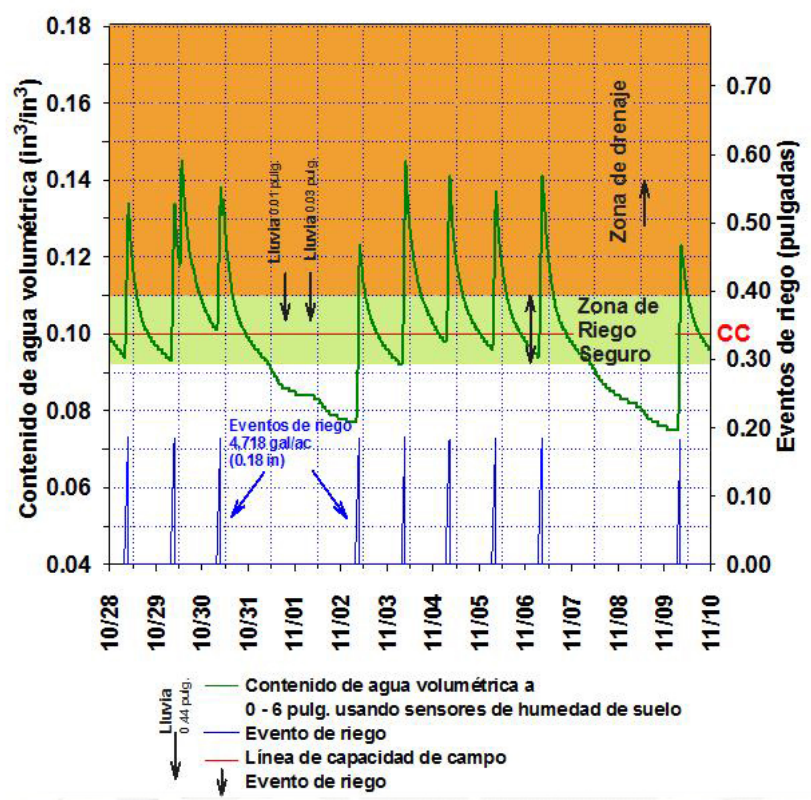

Figura 3. Ejemplo de un exceso de riego sobre el contenido volumétrico de humedad del suelo de 0-6 pulgadas de profundidad bajo las condiciones de cobertura vegetal de plástico para suelos arenosos.

producir un aumento significativo de la humedad del suelo. El 10/29, justo después del riego, se produjo un evento de lluvia fuerte de 0.44 pulgadas (in), el cual provocó un segundo aumento del contenido de humedad del suelo, el mismo día. El riego del día siguiente, (10/30) comenzó cuando el contenido volumétrico de agua del suelo se encontraba por encima de la capacidad del campo y pudo ser evitado sin ningún riesgo.

Entre el 10/31 y el 11/02, no se regó el cultivo. El contenido volumétrico de agua disminuyó de 0.14 a $0.08 \mathrm{pul}^{3} / \mathrm{pul}^{3}$. Debido a la baja capacidad de retención en los suelos arenosos, la falta de riego durante varios días podría resultar en un estrés hídrico innecesario para el cultivo, especialmente durante días muy calurosos o con mucho viento (altas tasas de evapotranspiración), o durante el período de crecimiento. Entre el 11/02 y el 11/06, se repitieron eventos de riego de gran magnitud, excediendo la "zona de riego segura," y produciendo un alto drenaje y lixiviación lixiviación de nutrientes.

Por lo contrario, la Figura 4 muestra un ciclo de riego "adecuado" para un período de 10 días. En este caso, el evento de riego comenzó exclusivamente cuando el contenido volumétrico de agua del suelo alcanzó un límite arbitrario. Para este caso en particular, se conocía la capacidad de campo, los eventos de riego comenzaron cuando el contenido de humedad del suelo alcanzaron valores por debajo de la capacidad de campo (por ejemplo, $0.09 \mathrm{in}^{3} / \mathrm{in}^{3}$ ). Sin embargo, para mantener el contenido volumétrico del 


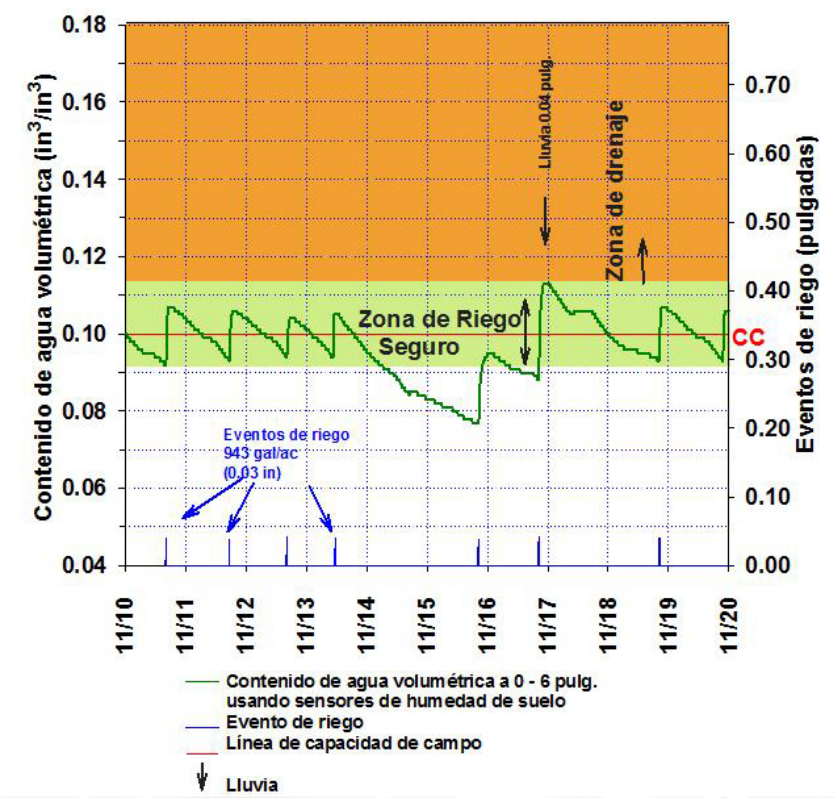

Figura 4. Ejemplo de una planificación adecuada de riego bajo condiciones de acolchado plástico en suelos arenosos con un contenido volumétrico de humedad comprendido entre 0-6 pulgadas.

agua en la "zona de riego segura," fue necesario hacer una determinación previa de la dosis de riego, para evitar un riego excesivo (se puede encontrar información adicional sobre dosis de riego en la publicación Microirrigation in Mulched Bed Production Systems: Irrigation Depths at http://edis.ifas.ufl.edu/ae049). La dosis de riego para la Figura 4 fue de 0.03 pulgadas por evento $(13 \mathrm{gal} / 100$ pies en riego por goteo), esta profundidad de riego fue suficiente para aumentar el contenido volumétrico de agua a una humedad específica, sin exceder la "zona de riego segura." En promedio, el contenido volumétrico de agua del suelo se encuentra próximo a la capacidad de campo, manteniendo el agua y los nutrientes dentro de la zona de las raíces. Para este ejemplo en particular, no se produjo percolación profunda de agua. Además, con la información del contenido del agua de suelo, el operario de riego puede tomar decisiones de no llevar a cabo el riego cuando el contenido de humedad del suelo se encuentre en un nivel adecuado. Por ejemplo, el 11/17, debido a un evento de lluvia de 0.04 pulgadas, no hubo necesidad de regar, debido a que la humedad del suelo estaba por encima de la capacidad de campo, por lo tanto, para mantener altos niveles de eficiencia no se realizó el riego.

\section{Resumen}

Este documento resume las directrices para la determinación de la capacidad de campo y la programación óptima del riego para suelos arenosos utilizando sensores de medición de la humedad del suelo (SHS). Los sensores de humedad del suelo han demostrado potencial para el monitoreo de la humedad del suelo, y para el respaldo en la toma de decisiones de riego en cultivos hortícolas. En el pasado, los sensores de humedad del suelo no eran ampliamente utilizados por los productores agrícolas debido a sus costes, al nivel técnico requerido y el mantenimiento de los sensores necesario. Los avances técnicos en los sensores de humedad del suelo han hecho que los mismos sean más fáciles de utilizar. Las restricciones continuas que apuntan a la disminución de lixiviación de nutrientes y los recientes aumentos en los costes de la energía han aumentado el interés en el uso de mejores tecnologías. Esta información se presenta para facilitarle al operario de riego la planificación y operación del sistema a un nivel óptimo. Con una planificación y operación adecuada, los sistemas de microriego pueden aplicar la cantidad precisa de agua al cultivo a unos altos niveles de eficiencia para alcanzar una máxima eficacia.

\section{Referencias}

Gary A. Clark and Dorota Z. Haman. 1988. Microirrigation in Mulched Bed Production Systems: Irrigation Depths. Pagina de Resultados AE72 del Dept. de Ing. Agr. Y Bio., Universidad de Florida. http://edis.ifas.ufl.edu/ae049. Ultima consulta 14 Enero 2013.

Muñoz-Carpena, R. 2004. Field Devices for Monitoring Soil Water Content. Extension Bul. 343 of the Dept. of Agr. and Bio. Engineering, University of Florida. http://edis.ifas.ufl. edu/ae266. Ultima consulta 14 Enero 2013. 